ISSN 0103-8478

\title{
Consumo de mesofilo foliar por Tuta absoluta (Meyrick, 1971) (Lepidoptera: Gelechidae) em três cultivares dE Lycopersicon esculentum Mill
}

\author{
Leaf mesophyll consumption by Tuta absoluta (Meyrick, 1971) \\ (Lepidoptera: Gelechidae) in three cultivars of \\ Lycopersicon esculentum Mill
}

Paulo Cesar Bogorni ${ }^{1}$ Ricardo Adaime da Silva² Gervásio Silva Carvalho $^{3}$

\section{RESUMO}

\begin{abstract}
A "traça-do-tomateiro", Tuta absoluta (Meyrick) (Lepidoptera: Gelechiidae), é uma das principais pragas do tomateiro na atualidade. Suas larvas atacam folhas, hastes, brotos, flores e frutos, causando severos danos à cultura. Trabalhos de quantificação do dano são relativamente raros $e$ restringem-se à contagem do número de frutos atacados $e$ estimativa dos danos foliares, nos testes de controle químico. Desta forma, realizou-se este trabalho objetivando quantificar o consumo de mesofilo foliar por este inseto em três cultivares de tomateiro (Carmem, Santa Clara e Empire). No Laboratório de Entomologia, do Departamento de Fitossanidade da Faculdade de Agronomia da Universidade Federal do Rio Grande do Sul, foi realizada a criação de lagartas nas três cultivares de tomateiro, sob condições controladas (temp. $25 \pm 1{ }^{\circ} \mathrm{C}$; U.R. $65 \pm 10 \%$ e fotofase de $12 \mathrm{~h}$ ). Através do uso de um medidor de área foliar (Li-cor Model LI-3000) mediu-se a área de mesofilo foliar consumida, correspondente ao dano em cada um dos ínstares. Não foram observadas diferenças no consumo de mesofilo foliar nas três cultivares. O consumo no $4^{\circ}$ instar foi significativamente superior aos demais, chegando
\end{abstract}

na cultivar Santa Clara, a mais consumida, a 2,207 $\pm 0,258 \mathrm{~cm}^{2}$, valor equivalente a $78,9 \%$ do total consumido durante a fase larval.

Palavras-chave: traça-do-tomateiro, consumo foliar, toma teiro.

\begin{abstract}
"Tomato leafminer", Tuta absoluta (Meyrick) (Lepidoptera: Gelechiidae), is one of the main pests of tomato crop nowadays. Its larvae attack leaves, stems, sprouts, flowers and fruits, causing severe damage to the crop. Reports on damage are quite rare and restricted to counting the attacked fruits and estimating the leaf damage in same papers about chemical control. Thus, this report aimed at quantifying the $T$. absoluta consumption in three tomato cultivars (Carmem, Santa Clara and Empire). Larvae were bred in three tomato cultivars at the "Laboratório de Entomologia do Departamento de Fitossanidade da Faculdade de Agronomia da Universidade Federal do Rio Grande do Sul", under controlled conditions (temp. $25 \pm 1{ }^{\circ} \mathrm{C} ;$ R.H. $65 \pm 10 \%$ and photophase of $12 \mathrm{~h}$ ). The
\end{abstract}

'Engenheiro Agrônomo, Mestre em Fitotecnia, Doutorando em Ciências (Entomologia Agrícola), Departamento de Entomologia, Fitopatologia e Zoologia Agrícola, Escola Superior de Agricultura "Luiz de Queiroz", Universidade de São Paulo, Av.: Pádua Dias, 11, CP 19, 13418-900, Piracicaba, SP. E-mail pcbogorn@carpa.ciagri.usp.br. Autor para correspondência.

${ }^{2}$ Engenheiro Agrônomo, Mestre em Fitotecnia, Doutorando em Agronomia (Entomologia Agrícola), Faculdade de Ciências Agrárias e Veterinárias, Universidade Estadual Paulista, Jaboticabal - SP. E-mail: rica@fcav.unesp.br

${ }^{3}$ Biólogo, Doutor, Professor Adjunto, Departamento de Fitossanidade, Faculdade de Agronomia, Universidade Federal do Rio Grande do Sul, Porto Alegre - RS. E-mail: gerve@vortex.ufrgs.br 
damaged area was measured in each one of all instars using a Portable Area Meter (Li-cor Model LI-3000). There were no differences in the leaf mesophyll area consumed among cultivars. The leaf consumption in the fourth instar was significantly

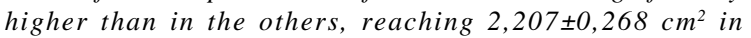
Santa Clara cultivar, the most consumed one, this value equals $78,9 \%$ of the whole consumption during the larval phase.

Key words: leafminer, leaf consumption, tomato.

\section{INTRODUÇãO}

Tuta absoluta (Meyrick,1917) (Lepidóptera: Gelechiidae), conhecida como a "traça-do-tomateiro",é uma das principais pragas da cultura do tomateiro (Lycopersicon esculentum Mill), na grande maioria dos países sul-americanos. Seu dano nas folhas se caracteriza por perfurações provocadas pelas larvas em forma de galeria, nas quais as mesmas se alimentam do mesofilo foliar, deixando apenas as epidermes, sendo possível, assim, observar regiões transparentes nas folhas, nos últimos ínstares larvais (BAHAMONDES \& MALLEA, 1969). Além das folhas, são atacados as brotações novas, flores e frutos (COELHO \& FRANÇA, 1987; HAJI et al., 1989; FERNANDEZ \& MONTAGNE, 1990; UCHÔA-FERNANDES etal., 1995).

No Brasil, o primeiro registro da praga foi em Morretes, no litoral paranaense, em setembro de 1979 (MUSZINSKI et al., 1982). Já em outubro do ano seguinte, sua ocorrência foi registrada em Jaboticabal, SP (MOREIRA et al., 1981), e em 1981 no Vale do Salitre em Juazeiro, BA (MORAES \& NORMANHA FILHO, 1982). Hoje, esta praga encontra-se distribuída por todas as regiões produtoras de tomate do Brasil.

Em relação à quantificação dos danos causados por este inseto, são raras as pesquisas desenvolvidas, sendo que, de uma forma geral, essas se restringem aos trabalhos com vistas ao controle, nos quais os danos são quantificados para análise da eficiência de produtos químicos ou de outros métodos. Na grande maioria dos trabalhos, registra-se o número de frutos atacados, independente da quantidade de larvas no seu interior, pois a ocorrência de uma única perfuração já deprecia o fruto (MOORE, 1983; HAJI et al. 1995). Um dos poucos trabalhos que consideraram o dano foliar, porém, não o quantificando, foi realizado por LOURENÇÃO (1984), utilizando uma estimativa visual do percentual de área foliar consumida para identificação de genótipos de tomateiro resistentes ao inseto.

Com o objetivo de quantificar os danos causados por T. absoluta às folhas de tomateiro, foi realizado este trabalho que comparou o consumo de mesofilo foliar por larvas nos diferentes ínstares em três cultivares de tomateiro sob condições de laboratório.

\section{MATERIALEMÉTODOS}

Este trabalho foi desenvolvido no Laboratório de Entomologia do Departamento de Fitossanidade da Faculdade de Agronomia da Universidade Federal do Rio Grande do Sul. Foram utilizadas as cultivares Santa Clara, Empire e Carmen, pertencentes aos tipos quadrado, plurilocular e longa-vida, respectivamente (as escolhas das cultivares se deram exclusivamente pela fenologia dos frutos, com um representante de cada um dos três grupo). Os insetos utilizados neste trabalho foram provenientes de três criações conduzidas em folhas de tomateiro, mantidas em laboratório no referido Departamento.

As lagartas foram acondicionadas em placasde-Petri ( $8 \mathrm{~cm}$ de diâmetro), com o fundo revestido com papel de filtro. Em cada placa, foi colocado um folíolo de tomateiro de aproximadamente $12 \mathrm{~cm}^{2}$, com o pecíolo envolto por algodão umedecido em água destilada para evitar a desidratação. Em cada folíolo, foi colocado um ovo da "traça-do-tomateiro" com três dias de idade, sendo que, para cada cultivar, foram utilizadas 40 placas, 10 para cada um dos quatro ínstares, onde cada placa perfazia uma repetição. As larvas foram criadas sob condições controladas, com temperatura de $25 \pm 1^{\circ} \mathrm{C}$, umidade relativa do ar de $65 \pm 10 \%$, e fotofase de 12 horas. As lagartas foram retiradas um pouco antes do final do ínstar, o qual foi definido através da mensuração diária do comprimento do corpo e da largura da cápsula cefálica. Estas medições foram feitas com o auxílio de uma ocular micrométrica acoplada a um microscópio-estereoscópio com uma fonte de luz na parte inferior, permitindo a visão da lagarta por transparência.

As lagartas de T. absoluta, ao se alimentarem do mesofilo foliar, deixam apenas as epidermes foliares. Após a retirada das larvas, estas epidermes remanescentes foram extraídas, sob microscópio-estereoscópio. Uma vez retiradas as epidermes no local do dano, a projeção deste foi hachurada em uma folha de papel sulfite branco. Posteriormente, efetuou-se uma fotocópia das áreas hachuradas em transparência e, com o auxílio de um medidor de área foliar (Portable Area Meter (Li-cor) Model LI-3000), foram mensuradas as áreas correspondentes aos danos.

As larvas foram mantidas nas folhas desde a fase de ovo até o final do ínstar, no qual se desejava avaliar o consumo de mesofilo foliar. Desta forma, o consumo no 2, $3^{\circ}$ e $4^{\circ}$ ínstares refere-se ao montante acumulado desde o início da fase larval. Assim, para a obtenção do consumo por ínstar foi subtraído o valor acumulado até o ínstar da avaliação pelo consumo médio acumulado nos ínstares anteriores. Foi calcula- 
do também, o consumo de mesofilo foliar diário em cada ínstar, baseado nas anotações da duração em dias dos ínstares do inseto individualmente.

Os testes foram desenvolvidos no delineamento experimental completamente casualizado em esquema fatorial com dois fatores (ínstares e cultivares) e 10 repetições. Os dados foram submetidos à análise de variância, sendo que para a comparação entre ínstares realizou-se a complementação da análise pelo teste de Tukey a 5\% de probabilidade de erro.

\section{RESULTADOS E DISCUSSÃO}

Os dados de consumo médio de mesofilo foliar por T. absoluta, por ínstar e acumulado por ínstar em cada cultivar, estão apresentados na tabela 1. Não houve diferença significativa no consumo de mesofilo foliar por ínstar ou acumulado nas cultivares (teste $\mathrm{F}$ a $5 \%$ de probabilidade de erro). Porém, verificou-se tendência de um maior consumo no $4^{\circ}$ ínstar, na cultivar Santa Clara. Esta tendência poderia ser parcialmente explicada pela duração do referido ínstar, que na cultivar Santa Clara foi de 3,6 dias, enquanto que nas cultivares Empire e Carmen foi de 3,4 e 3,1 dias respectivamente (Figura 1). Esta maior duração do 40 ínstar na cultivar Santa Clara refletiu num aumento da fase larval em relação às demais cultivares e conseqüentemente num maior período de consumo não só no último ínstar, como no acumulado.

No entanto, ao analisar os dados de consumo médio diário por instar nas três cultivares, apresentados na tabela 2 , verifica-se de fato que não apenas o aumento da fase larval influenciou no consumo na cultivar Santa Clara mas que, independentemente da duração da fase, houve maior consumo diário nesta cultivar pela praga, comprovando a tendência inicialmente evidenciada.

Observou-se que, no 4 o ínstar, os insetos apresentam o maior consumo diário, sendo significativamente diferente dos demais, independente da cultivar da qual se alimentaram (Tabela 2).
Ao observar o consumo relativo (Figura 2), verifica-se um alto percentual de consumo de mesofilo foliar no 4 i ínstar, chegando a 78,9\% do total acumulado na fase larval, na cultivar Santa Clara, valor equivalente a $2,207 \mathrm{~cm}^{2}$ consumidos por larva durante o ínstar (Tabela1).

O fato de as lagartas terem ingerido $3 / 4$ do total da área consumida durante a fase larval apenas no último ínstar, não pode ser explicado exclusivamente pelo aumento em seu tamanho, pois enquanto este segue tendência linear (VARGAS, 1970; QUIROZ, 1976; BOGORNI, 1999), o aumento no consumo de mesofilo foliar cresce em escala exponencial (Figura 2). Isto pode estar associado ao fato de o inseto ter necessidade de acumular reservas para as fases de pupa e adulta, fases estas de intenso metabolismo, e de reduzida ingestão de alimento (reduzida ingestão na fase adulta e nenhuma na fase de pupa).

O potencial de dano desta praga é comprovado não somente pelo seu ciclo relativamente curto, como também pelo grande consumo individual das lagartas, sobretudo no último ínstar. Isto sugere trabalhos posteriores relativos à elaboração de níveis de dano e de controle da praga baseados no consumo foliar, que possam facilitar o seu manejo, diminuindo o número de aplicações de inseticidas e aumentando a eficiência de controle.

\section{CONCLUSÕES}

As cultivares de tomateiro não exercem efeito antibiótico e/ou antixenótico sobre a fase larval de $\boldsymbol{T}$. absoluta, o que resultou na não diferenciação no consumo de mesófilo foliar.

\section{REFERÊNCIASBIBLIOGRÁFICAS}

BAHAMONDES, L. A.; MALLEA, A R. Biologia en Mendoza de Scrobipalpula absoluta (Meyrick) Povolny (Lepidoptera Gelechiidae), especie nueva para la Republica Argentina. Revista de la Faculdad de Ciencias Agrarias, Mendoza, v.15, n.1, p.96-104, 1969.

Tabela 1 - Consumo médio de mesofilo foliar por Tuta absoluta nos diferentes ínstares em três cultivares de tomateiro (temp. $25 \pm 1^{\circ} \mathrm{C}$; U.R. $65 \pm 10 \%$ e fotofase de $12 \mathrm{~h})$.

\begin{tabular}{lccccc}
\hline \multirow{2}{*}{ Cultivares $^{2}$} & \multicolumn{5}{c}{ Área consumida por lagarta $\left(\mathrm{cm}^{2} \pm \mathrm{EP}^{1}\right)$} \\
\cline { 2 - 6 } & $1^{10}$ Ínstar & $2^{\text {o }}$ Ínstar & $3^{\circ}$ Ínstar & $4^{\text {o }}$ Ínstar & Acumulado \\
\hline Empire & $0,041 \pm 0,003$ & $0,100 \pm 0,008$ & $0,467 \pm 0,082$ & $1,611 \pm 0,264$ & $2,219 \pm 0,264$ \\
Santa Clara & $0,042 \pm 0,007$ & $0,115 \pm 0,014$ & $0,432 \pm 0,075$ & $2,207 \pm 0,258$ & $2,796 \pm 0,258$ \\
Carmen & $0,034 \pm 0,003$ & $0,138 \pm 0,017$ & $0,561 \pm 0,083$ & $1,519 \pm 0,297$ & $2,253 \pm 0,297$ \\
\hline
\end{tabular}

${ }^{1} \mathrm{EP}=$ erro padrão da média

${ }^{2}$ Não houve diferença significativa entre as médias de cultivares pelo teste de Tukey a 5\% de probabilidade de erro. 


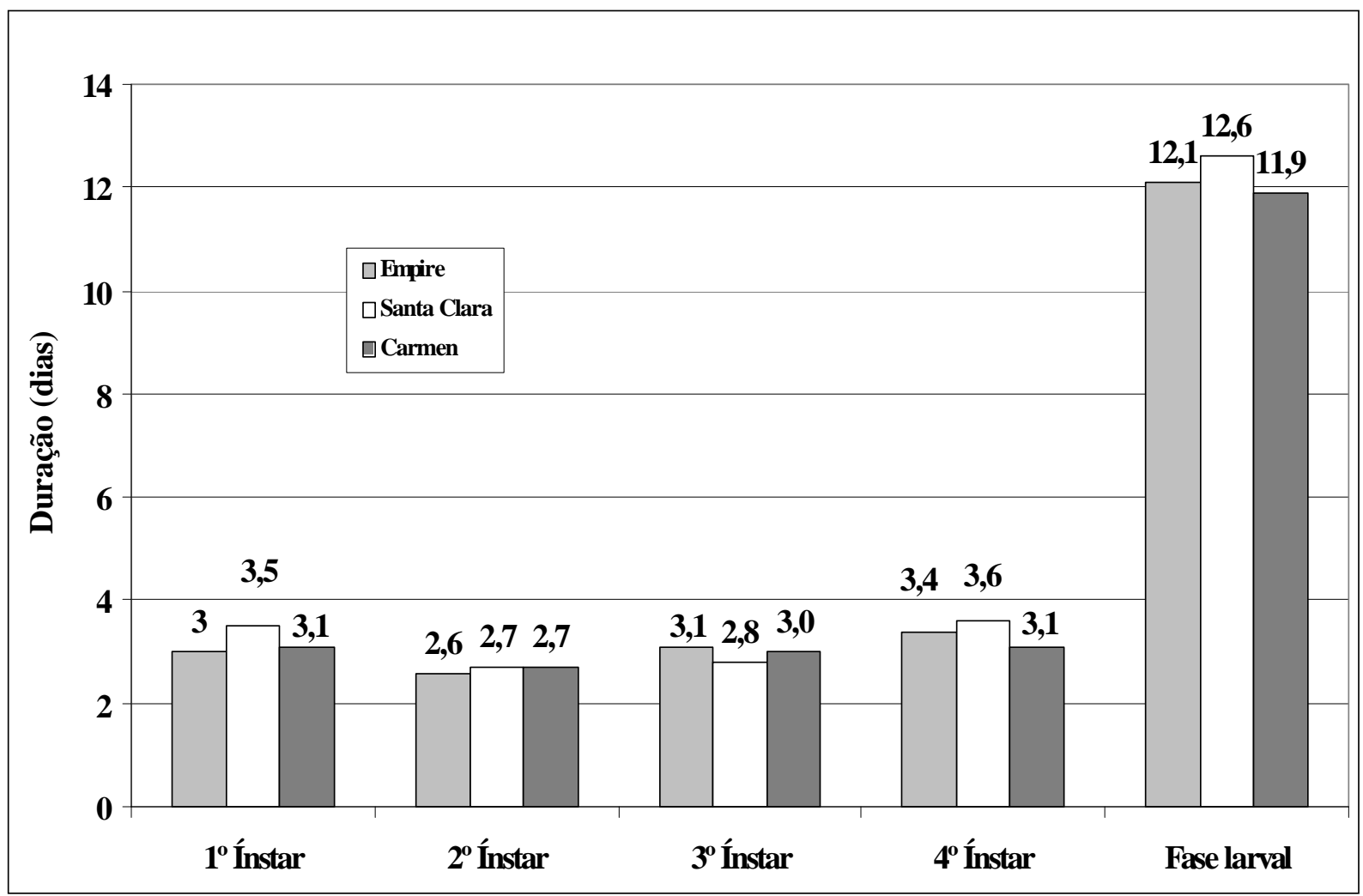

Figura 1 - Duração (dias) dos diferentes íntares de Tuta absoluta em três cultivares de tomateiro (temp. $25 \pm 1^{\circ} \mathrm{C}$; U.R. $65 \pm 10 \%$ e fotofase de 12h).

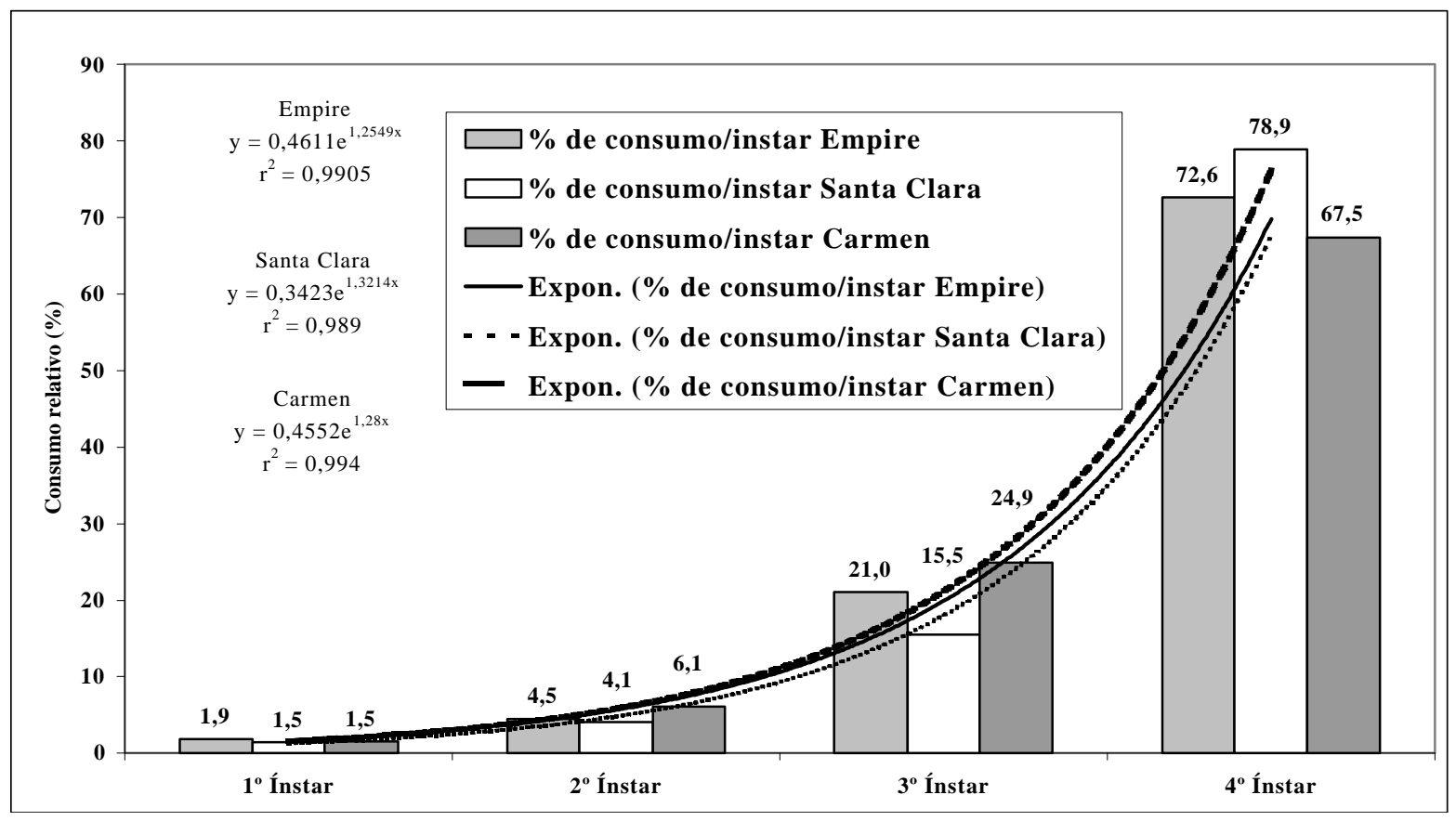

Figura 2 - Consumo relativo (\%) de mesofilo por Tuta absoluta em três cultivares de tomateiro (temp. $25 \pm 1^{\circ} \mathrm{C}$; U.R. $65 \pm 10 \%$ e fotofase de $12 \mathrm{~h}$ ) 
Tabela 2 - Consumo médio diário de mesofilo foliar por Tuta absoluta nos diferentes ínstares, em três cultivares de tomateiro (temp. $25 \pm 1^{\circ} \mathrm{C}$; U.R. $65 \pm 10 \%$ e fotofase de $12 \mathrm{~h}$ ).

\begin{tabular}{|c|c|c|c|}
\hline & \multicolumn{3}{|c|}{ Consumo médio $\pm \mathrm{EP}^{1}$} \\
\hline & Empire $^{2}$ & Santa Clara ${ }^{2}$ & Carmen $^{2}$ \\
\hline $1^{\circ}$ Ínstar & $0,014 \pm 0,001 \mathrm{a}$ & $0,012 \pm 0,002 \mathrm{a}$ & $0,012 \pm 0,002 a$ \\
\hline $2^{-}{ }^{\circ}$ Ínstar & $0,039 \pm 0,012 \mathrm{a}$ & $0,044 \pm 0,005 \mathrm{a}$ & $0,050 \pm 0,005 a$ \\
\hline $3^{-0}$ Ínstar & $0,157 \pm 0,031 \mathrm{a}$ & $0,151 \pm 0,023 \mathrm{a}$ & $0,187 \pm 0,028 a$ \\
\hline $4^{-0}$ Ínstar & $0,475 \pm 0,067 b$ & $0,616 \pm 0,065 b$ & $0,498 \pm 0,101 b$ \\
\hline
\end{tabular}

${ }^{1} \mathrm{EP}=$ erro padrão da média

${ }^{2}$ Médias não seguidas de mesma letra na coluna diferem significativamente pelo teste de Tukey a $5 \%$ de probabilidade de erro.

BOGORNI, P. C. Biologia e consumo foliar de Tuta absoluta (Meyrick, 1917) (Lepidoptera: Gelechiidae) em diferentes cultivares de Lycopersicon esculentum Mill. 1999. 88f. Dissertação (Mestrado em Fitotecnia) - Programa de Pós-graduação em Fitotecnia, Universidade Federal do Rio Grande do Sul.

COELHO, M.C.F.; FRANÇA, F.H. Biologia, quetotaxia da larva e descrição da pupa e adulto da traça-do-tomateiro. Pesquisa Agropecuária Brasileira, Brasília, v.22, n.2, p.129$135,1987$.

FERNANDEZ, S.; MONTAGNE, A. Biología del minador del tomate, Scrobipalpula absoluta (Meyrick) (Lepidoptera: Gelechiidae). Boletín de Entomología Venezolana, Maracay, v.5, n.12, p.89-99, 1990.

HAJI, F.N.P.; DIAS, R. de C.S.; ANDRADE, M.W. de. Controle da traça do tomateiro. Petrolina : EMBRAPA/CPATSA, 1989. 2p. (Comunicado técnico, n.39)

HAJI, F.N.P. et al. Manejo integrado de Scrobipalpuloides absoluta (Povolny) (Lepidoptera: Gelechiidae) no Submédio São Francisco. Anais da Sociedade Entomológica do Brasil, Jaboticabal, v.24, n.3, p.587-591, 1995.

LOURENÇÃO, A.L. Fontes de resistência a Scrobipalpula absoluta (Meyrick, 1917) em tomateiro. Bragantia, Campinas, v. 43, n.2, p.569-577, 1984.

MOORE, J.E. Control of tomato leafminer (Scrobipalpula absoluta) in Bolívia. Tropical Pest Management, London, v.29, n.3, p.231-238, 1983 .

MORAES, G.J. de; NORMANHA FILHO, J.A. Surto de Scrobipalpula absoluta (Meyrick) em tomateiro no trópico semi-árido. Pesquisa Agropecuária Brasileira, Brasília, v.17, n.3, p.503504, 1982.

MOREIRA, J.O.T.; LARA, F.M.; CHURATA-MASCA, M.G. C. Ocorrência de Scrobipalpula absoluta (Meyrick) (Lepidoptera, Gelechiidae) danificando tomate rasteiro em Jaboticabal, SP. In: CONGRESSO BRASILEIRO DE ENTOMOLOGIA, 7., 1982, Fortaleza. Resumos... Fortaleza : SEB, 1981. p.58.

MUSZINSKI, T.; LAVENDOWSKI, I.M.; MASCHIO, L.M. de A. Constatação de Scrobipalpula absoluta (Meyrick, 1917) (=G norimoschema absoluta) (Lepidoptera: Gelechiidae), como praga do tomateiro (Lycopersicon esculentum Mill.) no litoral do Paraná. Anais da Sociedade Entomológica do Brasil, Jaboticabal, v.11, n.2, p.291-292, 1982.

QUIROZ, E.C. Nuevos antecedentes sobre la biologia de la polilla del tomate, Scrobipalpula absoluta (Meyrick). Agricultura Técnica, Santiago, v. 36, n.2, p.82-86, 1976.

UCHÔA-FERNANDES, M.A.; DELLA LUCIA, T.M.C.; VILELA, E.F. Mating, oviposition and pupation of Scrobipalpuloides absoluta (Meyr.) (Lepidoptera: Gelechiidae). Anais da Sociedade Entomológica do Brasil, Jaboticabal, v.24, n.1, p.159-164, 1995.

VARGAS, H. Observaciones sobre la biologia y enemigos naturales de la polilla del tomate. Gnorimoschema absoluta (Meyrick), (Lep. Gelechiidae). Idesia, Arica, v.1, p.75-110, 1970 . 